\title{
Foreword
}

\section{Preparative chromatography today}

\author{
H. Colin
}

Prochrom S.A., 5 rue Jacques Monod, BP. 9, 54250 Champigneulles, France

reparative chromatography was for a long time considered by production engineers as a very expensive and unefficient purification technique, basically not usable for industrial size purification. Actually, most of these engineers were trying everything possible to avoid using chromatography. They were right in the sense that preparative chromatography was traditionally associated with low efficiency columns packed with large particle size packing materials, unstable and with poorly reproducible performance. Large quantities of solvents were required and the purified products were recovered in a (very) diluted form. In addition, there was no theoretical framework to understand phenomena related to column overloading and to model separations. "Optimisation" of operating conditions was more based on feelings and habits than on scientific facts. All these reasons certainly justified the bad feeling of production engineers about preparative chromatography. This was a very unfortunate situation, however, because it was recognised that chromatography was (and still is, obviously) one of the most efficient separation techniques. This situation started to change about $10-15$ years ago when the technologies of compression columns became available (radial and particularly axial compression) and when people realised that high quality columns (in terms of both hardware and packing material) could be used at large scale. This was the beginning of Modern High Performance Preparative Liquid Chromatography, also called PHPLC. Since its birth, PHPLC has seen a tremendous growth, particularly in the pharmaceutical industry. It is clear that PHPLC is used today by most pharmaceutical companies (and is beginning to be used by the fine chemical industries as well), both at the pilot scale level and also more and more at the production size level.

The de velopment of PHPLC has been made possible due to work in several areas: column technology, packing materials (both non-chiral and chiral), theory and modelling.

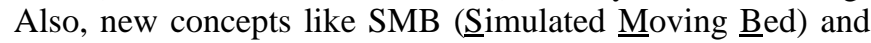
PSFC (Preparative $\underline{S}$ upercritical Fluid $\underline{\text { Chromatography) }}$ have been introduced to broaden the field of application of chromatography as an industrial purification tool. These various topics are addressed in the present dossier.

As for analytical chromatography, the heart of a preparative chromatograph is the column. This concerns both hardware and packing material. Column technology, and equipment in general, was the first step in the development of PHPLC since the most critical drawbacks of traditional preparative chromatography was the very poor column stability (this is obviously a very serious problem if one considers using chromatography as a production tool) and the low level of performance. The article of J. Dingenen examines the present situation of hardware. Compression columns, and more particularly columns based on the dynamic axial compression concept (DAC ${ }^{\mathrm{TM}}$ ) are an absolute necessity, to provide both stable and efficient columns that can be packed with small size particles. DAC columns also offer the user the possibility to pack himself/herself columns of adjustable length, simply and quickly, and with any kind of packing material. This gives great versatility and freedom, which is very valuable for industrial applications. Large columns, up to $1 \mathrm{~m}$ in diameter, rated at about 100 bar or more, are available today. They can be used for production capacities of several metric tons per year. They are designed

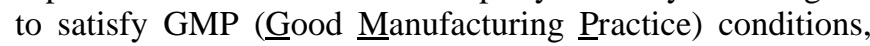
as required by relevant authorities.

The best hardware would be of no use without a good packing material. The most practical way to increase column efficiency (and thus reduces purification time, solvent consumption and product dilution, and then purification cost) is to use rather small particles. Compared to traditional preparative chromatography, PHPLC uses much smaller particles, typically 10 to $20 \mu \mathrm{m}$ (compared to more than $100 \mu \mathrm{m}$ ), packed in shorter beds (typically 20 to $50 \mathrm{~cm}$ long compared to more than $1 \mathrm{~m}$ ). In addition, it is also important that packing materials have a narrow size distribution, in order to avoid excessive column back pressure because of fine particles (pressure is expensive at large column size) and reduced efficiency because of large particles. These considerations have pushed packing material manufacturers to offer such small size packing materials with narrow size distribution, in large quantities, with satisfactory batch to batch reproducibility and at acceptable prices. In this respect, incidentally, it is worth mentioning that, although the best materials are still quite expensive, the contribution of the packing material to the total purification cost is often less than $15-$ $20 \%$. Using a low-quality/low-price material instead of a high-quality/medium-to-high-price one can often make the purification more expensive, in fact. Columns for PHPLC have now performance levels which are similar to those of analytical columns. This results in smaller solvent consumption, shorter purification times, more reproducible results and more economical separations. Properties of packing materials (except those for chiral separations) are discussed in the article of D. Sanchez.

The behaviour of chromatographic columns under overloading conditions is quite a complex situation. Weird effects can happen (and often do) when the columns are heavily overloaded, making preparative chromatograms somehow confusing. Before theoretical work was available to describe phenomena associated with column overloading, adjustment of operating conditions was quite empirical and many purifications were carried out under conditions far away from being optimised. Results were, accordingly, quite 
disappointing and preparative chromatography was claimed to be unappropriate for industrial uses. Actually, chromatography itself was not really to blaim because the disappointing results were due to the choice of improper operating conditions. Theoretical models have made the behaviour of overloaded columns much more understandable.

Such phenomena as displacement and tag-along effects have been understood, as well as the effects of column efficiency, mobile phase flow rate, column design, etc. An important step was to realise that columns can be much more overloaded than often though. Another important step was to recognised that preparative columns must be efficient. However, the theory of overloaded chromatography is quite complex and not readily accessible to anyone, be that only because calculation programs are required in order to use the theoretical framework. In this respect, simulation software is a very precious help, and, even if optimum purification conditions cannot be precisely calculated, trends are clearly shown by simulations. The contributions of A. Katti and Per Jagland, and A. Seidel-Morgenstern discuss theory and modelling/simulation aspects and address various ways to run chromatographic processes.

The interest of the industrial world for chromatography as a purification technique has certainly been a good insentive to push equipment manufacturers to develop new chromatographic techniques and equipment. The most exiting ones today are probably SMB and PSFC. The situation of PSFC is described in the article of P. Jusforgues and M. Shaimi. Supercritical fluids combine, to first approximation, the solvation properties of liquids (they are "good" solvents) and the kinetic properties of gases (their viscosity is small and diffusion coefficients are accordingly large). This makes such fluids very attractive for chromatographic purposes, and more particularly for preparative applications. In some ways, PSFC is quite similar to PHPLC: the columns (hardware and packing material) are basically the same and retention phenomena are also similar (displacement/tagalong effects, etc.). Only the mobile phase is different: a supercritical fluid instead of a liquid. In reality, PSFC and HPLC are quite different. The most commonly used supercritical fluid, by far, is carbon dioxide. It is safe for the operator and the environment, easy to obtain under supercritical conditions and cheap. This greatly militates for its use in a purification process. In addition, it is easy to eliminate the mobile phase in the collected fractions: a simple depressurisation (i.e. to atmospheric pressure) is sufficient to transform super-critical carbon dioxide into a gas. This is obviously of great interest for industrial applications. In this respect, implementation of mobile phase recycling is quite simple (in theory at least!): it is only necessary to recover the carbon dioxide in gas phase and, after having cleaned it, to put it back under supercritical conditions. This possibility of easy recycling of carbon dioxide combined with the low cost of this material makes PSFC very attractive for industrial purification, particularly if one keeps in mind the fact that the largest contribution to the cost of a purification process by PHPLC is the mobile phase (even when it is recycled). However, PSFC has some drawbacks as well: the technology is rather expensive (the equipment must be rated to several hundreds of bars), the manipulation of a supercritical fluid is not as simple as that of a liquid and the solvation properties of carbon dioxide do not allow to solubilise all products. This is particularly true for large and/or very polar molecules. The addition of a modifier often helps, but it partly eliminates at the same time some of the advantages outlined above. Nobody is perfect...

SMB is another very interesting and powerful technique to purify compounds. Although its principle is not new (huge quantities of sugars have been purified for many years in the food industry, for instance); its application to separation problems in the fine chemical and pharmaceutical industries is much more recent. This is partly due to the fact that a theoretical framework and simulation programs are now available. In addition, hardware aspects related to rather recent development of PHPLC (column technology, automation, etc.) have greatly contributed to the "rebirth" of SMB. J. Blehaut and R.M. Nicoud discuss the present situation of SMB. SMB is basically a binary separator (simulated liquidsolid counter-current process) allowing for the separation of a mixture in two fractions. Obviously, and depending on the crude composition, each fraction may contain more than one component. In order to simulate the counter-current process (simulate because it is difficult to move a solid phase and mass transfer phenomena in fluidised beds are not very efficient), the column is made of individual sections arranged in a circular way. Injection and collection points are moved along the columns caroussel. This is obviously an over-simplified description of an SMB system and great detail is given in the paper of Blehaut and Nicoud. The major advantages of SMB over PHPLC are basically: (1) the solvent consumption is often very reduced, (2) the collected products are less diluted, (3) the packing material is more efficiently used (this is a definite advantage when doing optical separations with chiral phases) and (4) the production rate per column volume unit is larger. Accordingly, purification costs are expected to be less with SMB than with PHPLC. Of course there are also problems and difficulties with SMB. One of them is related to the fact that SMB is a binary separator. Accordingly, if the product to be recovered is eluted in the middle of the chromatogram with products before and after, the purification needs to be done in more than one step. Also, solubility considerations may limit the production rate of an SMB unit. It must also be mentioned that the equipment is more sophisticated and probably more difficult to use (and maintain) than PHPLC equipment. Typically 8 to 16 columns are used (with very similar levels of performance) and the optimisation of purification conditions is more complex. Nevertheless, SMB is gaining popularity, particularly for large size purification, and there are certainly some excellent reasons to this. This is particularly the case for the resolution of racemic mixtures (a perfect example of binary separation), this topic being of very fast growing interest in the pharmaceutical industry.

Chiral separations are indeed becoming more and more common because authorities like the FDA (American Food and Drug Administration) have announced that a racemic mixture will no longer be accepted in the admittance test procedure for drugs in a near future. This has pushed manufacturers of packing materials to develop special stationary phases for chiral separations. G. Cox discusses this topic in his article. Cox examines the various types of materials available today (molecular imprinting, protein phases, cyclodextrin, etc.). The optimisation of enantioselective PHPLC is also discussed with particular emphasis on the selectivity (which is a key parameter in any chromatographic 
process). Some considerations are also made on the costs of chiral separations.

Finally, the contribution of G. Mann addresses what PHPLC can do for an engineer dealing with purification problems in the pharmaceutical industry. It is clear that this industry requires purer and purer products and that the purification problems are becoming more and more difficult. The use of PHPLC is not necessarily simple, however, because crude mixtures contain, in general, many by-products that can make the separation particularly difficult, unstable and expensive. "Tricks" have often to be used. A large number of options is available and techniques like PAU (PreAdsorption Units), precolumns, backflush, etc. help solving problems. There is no general chromatographic strategy, each separation problem being a specific case. If other and simpler techniques than PHPLC can be used, such as recrystallisation, for instance, then they should be used if they are cheaper. It is clear that, even if PHPLC often (always?) does the job of purifying, it is not the only technique available. It is not always obvious, however, to acertain that the other techniques are cheaper than PHPLC. Considerations like product purity and recovery yield must be compounded with the direct cost of the purification process. It must also be mentioned that time is becoming a more and more critical factor in the very competitive world of the pharmaceutical industry. PHPLC offers the advantage over other less powerful (and sometimes cheaper purification techniques) to work faster and to spend less effort on the synthesis conditions. And obviously, it makes a very big difference for a company to be first on the market, even if the production conditions of the relevant compound are not the most economical ones. 